\title{
THE HIERARCHICAL ORGANIZATION OF ARM STROKE IN A 400-M FREESTYLE SWIMMING RACE
}

original paper

( ) University School of Physical Education in Wroclaw

DOI: https://doi.org/10.5114/hm.2021.103284

\section{ANDREA MICHELE FREUDENHEIM ${ }^{1}$, FABRÍCIO MADUREIRA ${ }^{2}$, UMBERTO CESAR CORRÊA ${ }^{1}$}

${ }^{1}$ School of Physical Education and Sport, University of São Paulo, São Paulo, Brazil

${ }^{2}$ Faculty of Physical Education, Metropolitan University of Santos, Santos, Brazil

\begin{abstract}
Purpose. Arm stroke is a key variable of successful performance in front crawl swimming. In the present study, the effects of the arm stroke on the front crawl swimming performance were analysed by considering the complementarity of macroconsistency and micro-variability in the arm stroke as a hierarchically organized adaptive system. In this case, consistency is necessary to achieve outcomes reliably, and variability is fundamental for coping with environmental instability.

Methods. Displacements of swimmers $(n=31)$ who competed in the $400-\mathrm{m}$ freestyle race of the Paulista Master Championship were captured in 4 moments (partial races 1-4). From the aerial and aquatic phases of the left and right arm strokes, macrostructure (components' relative timing) and microstructure (components' overall time) had their variability rates calculated for all partial races on the basis of the biological coefficients of variation.

Results. It was revealed that swimmers: (i) increased consistency of macrostructure related to the left arm aerial phase stroke in the final race; (ii) maintained consistency of microstructure across races; and (iii) presented macrostructure with inferior rate of variability to the microstructure in the final race.

Conclusions. Given these results, coaches should emphasize instruction for swimmers to maintain the temporal relationship among arm stroke components (macrostructure) rather than focus on the components themselves (microstructure).
\end{abstract}

Key words: motor skill, performance, variability

\section{Introduction}

In the last few decades, there have been an increasing number of studies to understand the arm stroke as a key variable of successful performance in front crawl swimming [1-6]. As it is well known, the arm stroke is responsible for most of the propulsion in front crawl swimming [7-9].

In front crawl swimming, the arm stroke is characterized by a cycle formed by continuous and alternating circular movements of the left and right arms, in and out of the water. On the basis of Chollet's index of arm coordination [3], 3 main arm stroke cycles can be identified, which differ in terms of the beginning of the propulsive movement of one arm and the end of the propulsive movement of the other arm. These are: (a) opposition, when the end of the propulsive action of one arm occurs concomitant to the beginning of the propulsive action of the other arm; (b) catch-up, when there is a delay between the propulsive action of the 2 arms resulting in a negative percentage, i.e. during a period when the swimmer does not produce propulsion with either arm; and (c) superposition, when the index is positive, i.e. when there is simultaneous propulsive action of the 2 arms. It has been suggested that for elite swimmers of short and middle sprint races, the superposition coordination generates the largest crawl propulsion and, consequently, higher speed $[3-5,10,11]$.

Besides, several studies have shown how the arm stroke affects swimming performance through 2 main dimensions: spatial (stroke length [SL]) and temporal

Correspondence address: Andrea M. Freudenheim, Escola de Educação Física e Esporte, Universidade de São Paulo, Av. Prof. Mello Moraes, 65, Cidade Universitária, São Paulo - 05508-030, Brazil, e-mail: amfreud@usp.br

Received: October 18, 2019

Accepted for publication: May 22, 2020

Citation: Freudenheim AM, Madureira F, Corrêa UC. The hierarchical organization of arm stroke in a 400-m freestyle swimming race. Hum Mov. 2021;22(4):1-9; doi: https://doi.org/10.5114/hm.2021.103284. 
A. Freudenheim, F. Madureira, U. Corrêa, Arm stroke timing in front crawl swim

(stroke rate [SR]) [1, 2, 6, 12-14]. Generally, SL has been considered in meters per stroke. In turn, SR has been calculated by the number of strokes in a given time. Swimming speed $(v)$ has been determined as the product of those parameters and technically described by the following equation [11]:

$$
v=\mathrm{SL} \times \mathrm{SR}
$$

Numerous studies have indicated that the intraand inter-race variabilities of SL and SR play an important role in performance [1, 6, 13, 14]. Although such variabilities are dependent upon characteristics of swimmers and race speed, they also have been suggested to be a consequence of the swimmer's decision-making on adaptive strategies for dealing with constraints that emerge throughout the race $[1,3-5$, 13, 14].

Notwithstanding the advances provided by previous studies, as with any complex phenomenon, the role of the arm stroke concerning crawl swimming performance needs to be investigated from different perspectives and analysis levels. In the present paper, the analysis was based on an alternative view of variability in the motor skills as hierarchical adaptive systems [15]. Such a view allows advancing the existing knowledge by conceiving the complementarity of different levels of variability (e.g. macro- and microscopic) of a system (e.g. arm stroke), as well as the meaning of both in the performance as hallmarks of motor skill [15-19]. It is worth mentioning that, although arm stroke coordination (index of coordination [IdC]), SL, and SR play an important role in the crawl swimming performance $[1,6,13,14]$, they do not stand for the complementarity of different levels of variability (e.g. macro- and microscopic) of a hierarchical adaptive system (e.g. arm stroke).

Hierarchical adaptive systems, also named metastable open systems, are those systems in which the variability of the general pattern is significantly smaller than the variability of the components, and for this reason they present consistency of macroscopic behaviour and variability of microscopic behaviour [20-24]. This is the case with motor skills. In terms of motor skills, variability in the interaction of the components guarantees the general configuration of a pattern and, therefore, its consistency. In turn, the variability of components themselves is related to the performance options available within each one. Thus, variability of components themselves is responsible for pattern flexibility (i.e. parameterization) [15-18]. In this hierarchical adaptive system view of motor skills, the pattern consistency and component variability as dimensions of motor skills have been referred to as macro- and microstructures, respectively.

The macrostructure results from the interaction between components. It refers to the motor skill's overall pattern and, therefore, it is well-defined and order-oriented. Relative timing and sequencing have been recognized as invariant features of macrostructure. In turn, microstructure refers to the components themselves. It is ill-defined, variable, and disorderoriented. Absolute movement time and muscle group selection are some of the measures of microstructure [15-19]. For instance, the arm stroke emerges from the interaction between 4 main movement components: (a) right arm aerial phase; (b) right arm aquatic phase; (c) left arm aerial phase; and (d) left arm aquatic phase. Invariably, the arm stroke of front crawl swimming comprises the simultaneous execution of 2 components ( 1 and 4; 2 and 3 ) and sequential and continuous execution of these component dyads (1-4, 2-3...). In turn, the details of these simultaneities, such as the duration of each component, as well as the beginning time of each one, vary depending on the race context (e.g. 50-m vs. 400-m race; skilful swimmers vs. not skilled ones).

At the macrostructure level, the temporal and sequential relationship of the arms is well established. For example, while the right arm performs the aerial phase, the left arm performs the aquatic phase by considering the total stroke cycle time. On the other hand, at the microstructure level, each component can behave freely within certain limits established by the macrostructure. Thus, the aerial phase component of the right arm has a relative time that can be significantly invariable (macrostructure), although its absolute movement times may vary (microstructure).

On the basis of this view, we sought to investigate how variabilities of the arm stroke's macro- and microstructure would affect the performance of front crawl swimming. Considering the rate of macro- and microstructure variability change throughout a race [6], we focused on a 400-m freestyle race to allow for capturing the swimmers' performance in different moments. It was hypothesized that the fastest swimmers would show higher macro-consistence (i.e. lower variability rate) or higher micro-flexibility (i.e. higher variability) than other swimmers. Consequently, they would also present the macrostructure with a superior rate of variability compared with the microstructure variability of the components themselves. 


\section{Material and methods}

\section{Participants}

The participants were 31 swimmers aged 21-54 years $(M=32.8$ years, $S D=10.8)$, of these 21 males aged $21-54$ years $(M=34.1, S D=10.4)$ and 10 females aged $21-53$ years $(M=30, S D=11.5)$. They were highly skilled swimmers who participated in the 400-m freestyle race of an official Master Championship of Swimming of the São Paulo Aquatic Federation, Brazilian Confederation of Aquatic Sports. That is, all 31 participants, regardless of age, occupied in their respective series 1 of the 4 central lanes of the pool destined for the faster swimmers.

\section{Design and procedures}

All data collection procedures met the normative criteria of the Brazilian Confederation of Aquatic Sports. The swimmers' displacements were recorded by 4 digital cameras (Sony Cyber-shot, model DSC-V3) positioned $3 \mathrm{~m}$ above the edge opposite to that of starting, in front of the central lanes $(3,4,5$, and 6 swimming lanes).

The 400-m freestyle race was held in a semi-Olympic pool $(25 \mathrm{~m})$. Thus, the subjects swam 16 times the length of the pool. It is worth highlighting an original aspect of the study: it was carried out in a real competitive situation. By considering the race length, as a matter of methodological viability, the swimmers' displacements were captured in 4 moments: third, seventh, eleventh and fifteenth laps, hereinafter referred to as partial races $1,2,3$, and 4 , respectively. Besides, these laps were chosen because they were always in the same direction and did not include the starting (first lap) and the finishing (last lap), in which swimmers have to make adjustments regarding departure and arrival, respectively.

From the recording of swimmers' displacement, 3 stroke cycles performed over $10 \mathrm{~m}$ of the central pool (i.e. between the initial and final $7.5 \mathrm{~m}$ ) were selected for analysis through the VirtualDub 1.6 (GNU General Public License, Cambridge, MA, USA) software with a frequency of $60 \mathrm{~Hz}$. Although the front crawl swimming emerges from interaction among arm stroke, leg kick, body position, and breathing, this study focused only on the first one because it is strongly associated with speed in swimming [3, 25-28].

A stroke cycle was defined on the basis of the right arm's attack into the water. That is, a stroke cycle was considered from the moment the right arm attacked the water until it returned to the same position. This procedure allowed obtaining the time of aerial (considered from the moment the arm got out of the water until the attack) and aquatic (considered from the attack until the arm got out of the water) phases of both right and left strokes as stroke components. Measures of stroke cycle and stroke components made it possible to calculate the measures of macro- and microstructure of stroke patterns.

\section{Measures}

The macrostructure referred to the invariant stroke overall pattern, which emerged from the interaction of its components (C1 - right arm aerial phase; $\mathrm{C} 2$ right arm aquatic phase; C3 - left arm aerial phase; C4 - left arm aquatic phase). It was assessed on the basis of the relationship between each component and the stroke overall pattern. On the other hand, the microstructure measure referred to each components' behaviour and, therefore, reflected the variability in the stroke patterns. These structures were as follows:

\section{Macrostructure}

Relative time of right arm aerial phase (\%) was calculated as:

$$
\text { Rtra }=(\text { tra } \div \text { sc }) \times 100
$$

where Rtra was the relative time of right arm aerial phase, tra was the time of right arm aerial phase, and sc referred to the total time of stroke cycle.

Relative time of left arm aerial phase (\%) was calculated as:

$$
\text { Rtla }=(\text { tla } \div \text { sc }) \times 100
$$

where Rtla was the relative time of left arm aerial phase, tla was the time of left arm aerial phase, and sc referred to the total time of stroke cycle.

Relative time of right arm aquatic phase (\%) was calculated as:

$$
\text { Rtraq }=(\text { traq } \div \text { sc }) \times 100
$$

where Rtraq was the relative time of right arm aquatic phase, traq was the time of right arm aquatic phase, and sc referred to the total time of stroke cycle.

Relative time of left arm aquatic phase (\%) was calculated as: 


$$
\text { Rtlaq }=(\text { tlaq } \div \text { sc }) \times 100
$$

where Rtlaq was the relative time of left arm aquatic phase, tlaq was the time of left arm aquatic phase, and sc referred to the total time of stroke cycle.

\section{Microstructure}

From the values of each component of both strokes' macro- and microstructure in each cycle, the invariant and variable dimensions of the stroke pattern were assessed by calculating their rates of variability in the first, second, third, and fourth partial race. This was made by biological coefficients of variation:

$$
\mathrm{BCV}=\mathrm{CV} \%-\mathrm{SEM} \%
$$

where BCV stood for the biological coefficient of variation, CV was the rate of variability (coefficient of variation), and SEM was the standard error of the mean. CV was calculated as:

$$
\mathrm{CV}=(\sigma / \mu) \times 100
$$

where $\sigma$ referred to the standard deviation and $\mu$ was the arithmetic mean. SEM was obtained as:

$$
\mathrm{SEM}=\{[(\sigma / \sqrt{ } n) / \mu] \times 100\}
$$

where $\sigma$ referred to the standard deviation, $\mu$ was the arithmetic mean, and $n$ referred to the number of trials considered in the calculation.

The subtraction of SEM from CV has been used as a way of separating (filtering) the possible human errors in the capture of kinematic data, including data concerning swimming [6, 13, 29].

Furthermore, the performances in the race were obtained by an official timing and electronic scoreboard system of the local aquatic federation that were triggered by the swimmer's exit from the block and finalized by their touch on plates $(0.90 \mathrm{~m}$ high by $2.40 \mathrm{~m}$ long) attached to the pool wall.

\section{Statistical analysis}

To investigate the influence of the hierarchical organization of the front crawl stroke pattern on the performance of the 400-m freestyle swimming race, we analysed the macro- and microstructure on the basis of race times. They ranged from 265 to $531 \mathrm{sec}-$ onds and were divided in accordance with quartiles as the cut-off points [30]. In this case, the first quar- tile was the one with the fastest swimmers and the fourth quartile involved the slowest swimmers. This procedure occurred separately for male and female swimmers because their performances, as expected [31], were significantly different $(\mathrm{F}(1,29)=8.916, p=$ 0.006). In other words, male and female swimmers were grouped depending on the performance quartiles rather than the absolute values of performance.

To consider the interactions between performance quartiles and partial races, mixed-model ANOVAs $4 \times 4$ (performance quartiles $\times$ partial races) were conducted on data from each stroke component. Finally, by considering that in a hierarchically organized system the macro-variability is smaller than the microvariability, the macro- and microstructure were compared with each partial race by a mixed-model ANOVA $4 \times 2$ (performance quartiles $\times$ structure levels). In this case, the structures as a whole (overall macrostructure and overall microstructure) were considered through the arithmetic mean of the components' BCV. Both analyses were preceded by sex comparisons by oneway ANOVAs.

All observed significant effects were followed up by using Fisher $_{\text {HSD }}$ tests. All analyses were preceded by Shapiro-Wilk's $W$ and Bartlett's tests of normality and homogeneity of variance. All analyses were conducted with the Statistica 13.0 software (StatSoft, Tulsa, OK, USA) with the level of significance set at $\alpha=0.05$.

\section{Ethical approval}

The research related to human use has complied with all the relevant national regulations and institutional policies, has followed the tenets of the Declaration of Helsinki, and has been approved by the authors' institutional review board.

\section{Informed consent}

Informed consent has been obtained from all individuals included in this study.

\section{Results}

Figure 1 presents the BCV of the macro- and microstructure's components C1 (right arm aerial phase), C2 (right arm aquatic phase), C3 (left arm aerial phase), and C4 (left arm aquatic phase) of the first, second, third, and fourth performance quartiles of the swimmer groups. Regarding the macrostructure, it can be observed that the arm stroke's aerial phases had rates of variability superior to those in the aquatic phases. Also, throughout the partial races, the rates of vari- 


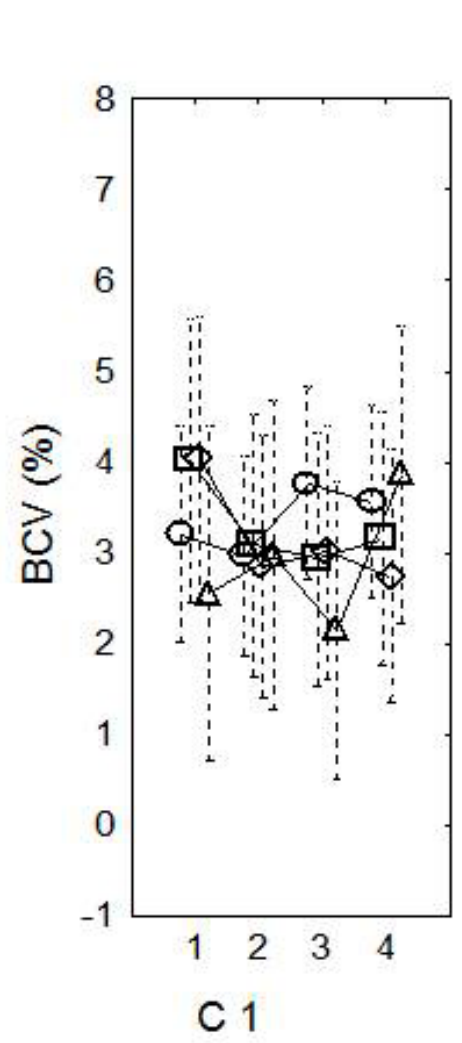

\section{MACROSTRUCTURE}
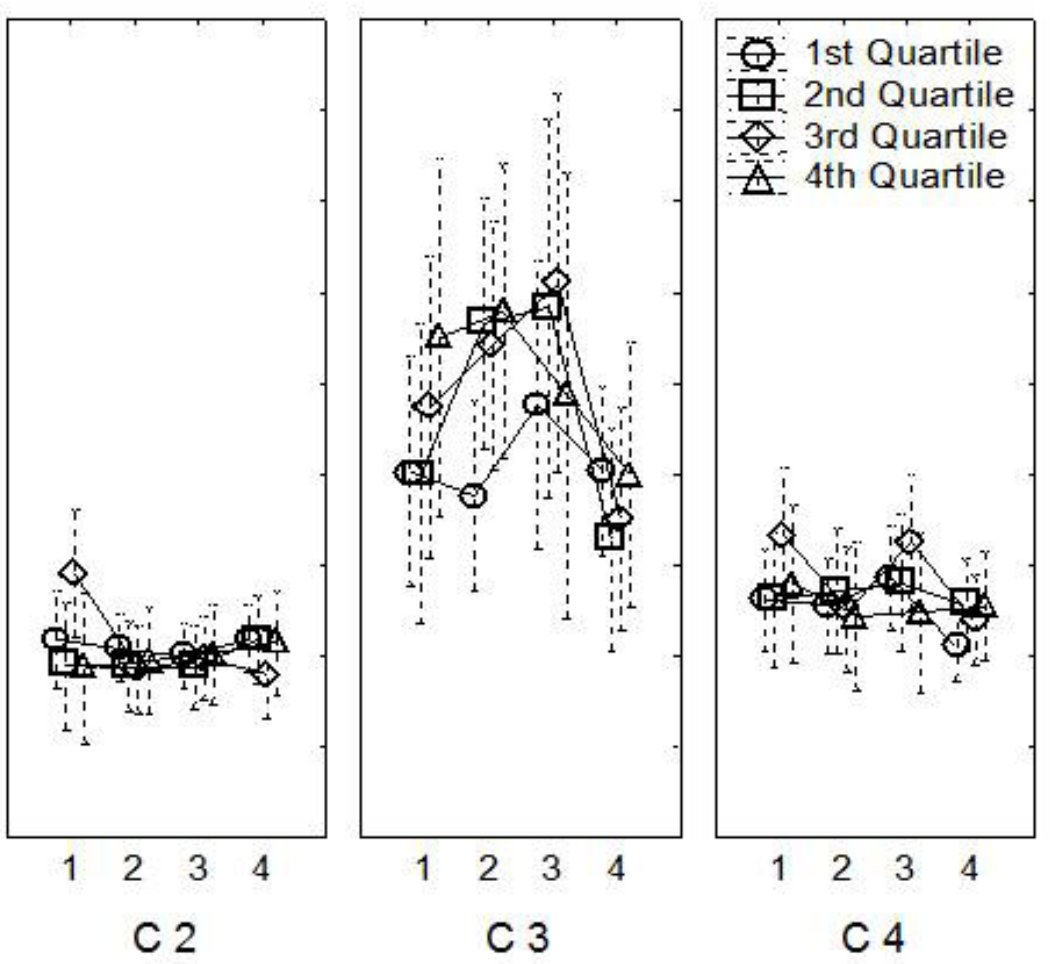

\section{MICROSTRUCTURE}
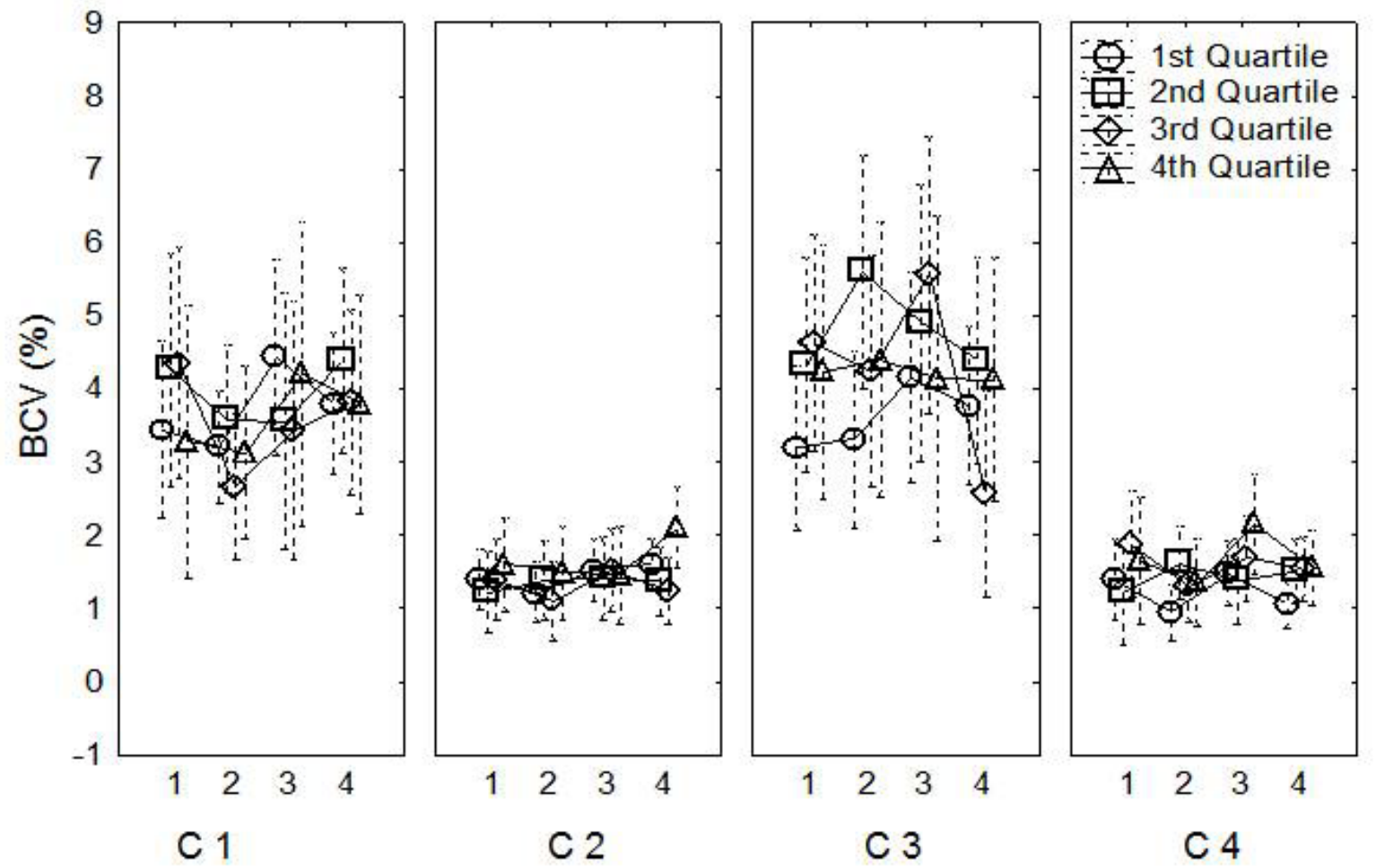

Figure 1. Biological coefficients of variation (BCV) of the macro- and microstructure's components $\mathrm{C} 1$ (right arm aerial phase), C2 (right arm aquatic phase), C3 (left arm aerial phase), and C4 (left arm aquatic phase) in the first, second, third, and fourth performance quartiles of swimmer groups 


\section{HUMAN MOVEMENT}

A. Freudenheim, F. Madureira, U. Corrêa, Arm stroke timing in front crawl swim
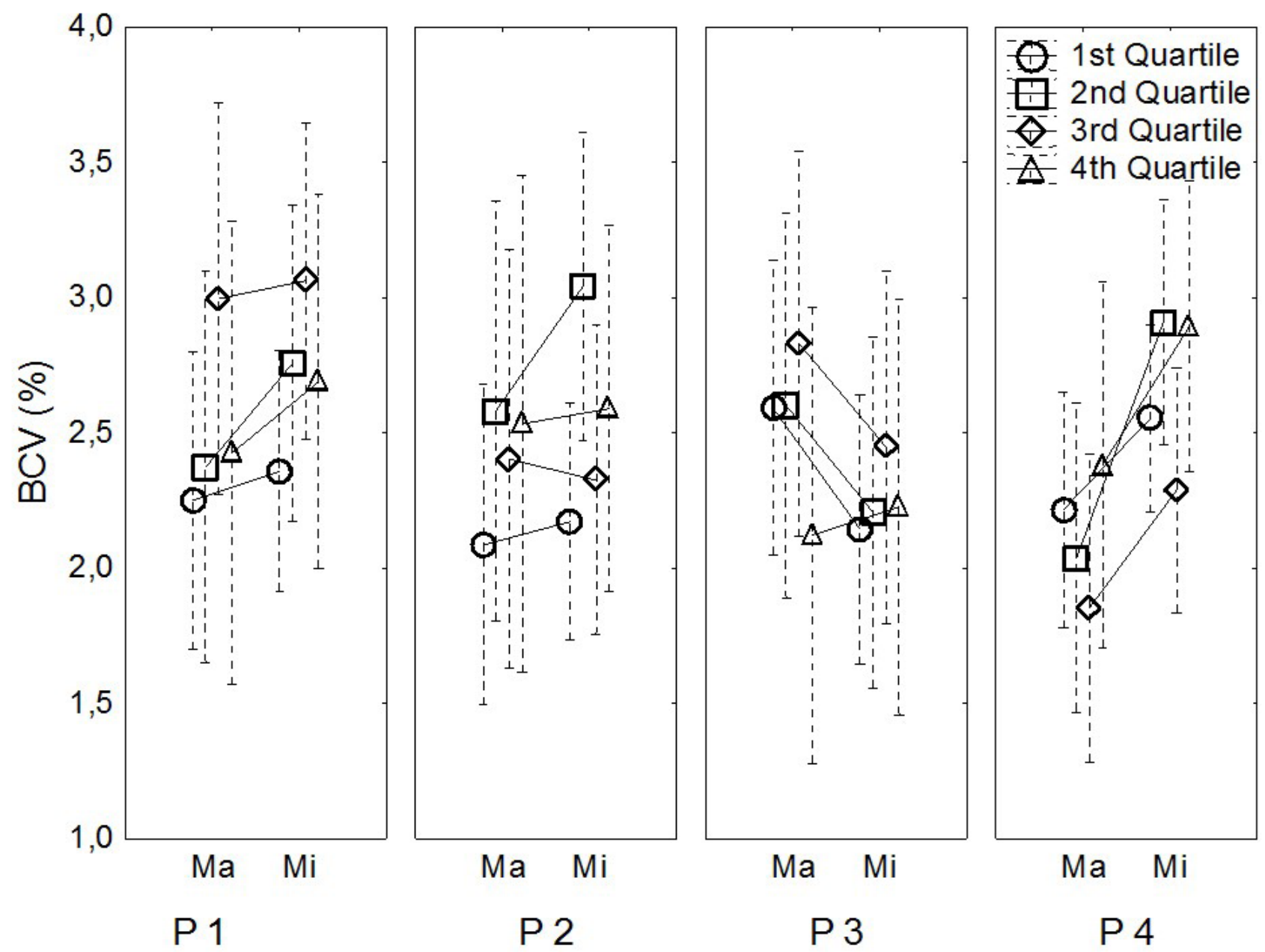

Figure 2. Biological coefficients of variation (BCV) of the macro- (Ma) and microstructure (Mi) in partial races (P) 1, 2, 3, and 4 of the first, second, third, and fourth performance quartiles of swimmer groups

ability of the aerial phases fluctuated more than those in the aquatic phases. Similar behaviour can be found for the microstructure.

Concerning macrostructure, no effects were revealed for sex comparisons. Mixed-model ANOVAs $4 \times 4$ (performance quartiles $\times$ partial races) determined effects only for partial races in $\mathrm{C} 3(\mathrm{~F}(3,81)=3.37, p=$ $0.002, \eta^{2}=0.11$ ). The Fisher ${ }_{\mathrm{HSD}}$ test showed that the fourth partial race had a lower rate of variability than the second $(p=0.039)$ and third $(p=0.005)$ partial races. This allows inferring that the left arm aerial phase became more consistent in the final part of the race regardless of the performance level. As for microstructure, no difference was revealed.

Concerning macro- vs. microstructure comparisons, Figure 2 presents the BCV of the macro- and microstructure in the partial races 1,2,3, and 4 of the first, second, third, and fourth performance quartiles of the swimmer groups. It can be noted that in most partial races, all performance quartile groups presented macrostructure with inferior variability rates com- pared with microstructure. This did not occur only in the third partial race. Furthermore, it appears that the third performance quartile group had a superior rate of variability in most partial races.

Similarly to the previous results, no effects were also revealed for sex comparisons here. A mixed-model ANOVA $4 \times 2$ (performance quartiles $\times$ structure levels) determined effects only for structure levels in the fourth partial race $\left(\mathrm{F}(1,27)=15.05, p=0.0001, \eta^{2}=\right.$ 0.45). The Fisher ${ }_{\mathrm{HSD}}$ test showed that macrostructure had an inferior rate of variability compared with microstructure $(p=0.0003)$.

\section{Discussion}

Total and relative times have long been used for assessing different dimensions of movement patterns, including inferring the role of the central nervous system on the variable (parameterization) and invariant (structure) aspects of movement patterns [32-35]. In the present study, both variable and invariant dimensions 
were assumed as levels of a single structure, that is, a complex system of hierarchical organization [15-19].

It was expected that the fastest swimmers would show a lower rate of variability in the macrostructure (invariant dimension) and, therefore, more consistency in the pattern structure, and a higher rate of variability in the microstructure (variant dimension) than other swimmers. Furthermore, assuming that in hierarchical adaptive systems, the variability of the general pattern is significantly smaller than the variability of each component [20-24], we hypothesized that the fastest swimmers would also show a superior variability rate in the macro- compared with the microstructure. These hypotheses are rooted in the prediction that macroconsistency and micro-variability allow an open system (i.e. arm stroke) to maintain its identity and adapt itself to environmental demands, respectively [22].

These hypotheses were partially confirmed. On the one hand, the results did not show the foregoing expected differences among groups. This was because all groups similarly changed their movement pattern in terms of the left arm aerial phase as a macrostructure component, making it more consistent in the final partial race. Why does this change occur? Perhaps this is related to how athletes distribute work and energy throughout an exercise task (strategy). As it is known, athletes self-select intensity and optimal pacing strategy by their ability to resist fatigue (i.e. anaerobic and aerobic supply) [36]. Additionally, there is a key characteristic of the best swimmers in maintaining a consistent SR over a 100-m race [12]. Specifically, concerning the results of the present study, 400-m freestyle swimmers are characterized by increasing consistency of arm stroke in the final partial race.

Another important question is: why are groups similar in making this change? We think this is related to the other results' explanatory hypothesis. As expected, results showed that all performance groups presented a similar macrostructure with an inferior rate of variability compared with microstructure in most partial races. We believe that this makes sense because all of the investigated swimmers were skilful. As indicated, we collected data for swimmers in the central lanes $(3,4,5$, and 6$)$, therefore involving the fastest swimmers. Thus, they may have a similar level of capability. The maintenance of pattern identity simultaneous with adaptability to environmental requirements refers to a characteristic of skilful behaviour for swimmers at the national and international performance levels [37]. Besides, the behaviour of the left arm aerial movement should be re-investigated to guarantee the consistency of results.

\section{Conclusions}

In summary, the findings of this study allowed us to conclude that 400-m freestyle race swimmers performed more consistently in the left arm aerial phase in the final partial race and that they showed an inferior rate of variability for the macrostructure compared with the microstructure.

As it is widely recognized, an athlete's pacing and arm stroke organization can have a significant impact on performance. On the basis of our findings, swimming coaches and teachers should instruct swimmers to maintain the temporal relationship among the arm stroke components (macrostructure) rather than the components themselves (microstructure). Regarding this suggestion, 2 aspects deserve to be highlighted: (i) our findings were obtained from a real competition context with elite athletes; (ii) the results had a moderate power of generalization, which reinforces the possibility of providing useful insights for the design of practice tasks in swimming.

\section{Disclosure statement}

No author has any financial interest or received any financial benefit from this research.

\section{Conflict of interest}

The authors state no conflict of interest.

\section{References}

1. Alberty MR, Potdevin FP, Dekerle J, Pelayo PP, Sidney MC. Effect of stroke rate reduction on swimming technique during paced exercise. J Strength Cond Res. 2011;25(2):392-397; doi: 10.1519/JSC.0b013e3181b $94 \mathrm{a} 51$.

2. Arellano R, Brown P, Cappaert J, Nelson RC. Analysis of 50-, 100-, and 200-m freestyle swimmers at the 1992 Olympic Games. J Appl Biomech. 1994;10(2):189-199; doi: 10.1123/jab.10.2.189.

3. Chollet D, Chalies S, Chatard JC. A new index of coordination for the crawl: description and usefulness. Int J Sports Med. 2000;21(1):54-59; doi: 10.1055/s-2000 $-8855$.

4. Millet GP, Chollet D, Chalies S, Chatard JC. Coordination in front crawl in elite triathletes and elite swimmers. Int J Sports Med. 2002;23(2):99-104; doi: 10.1055/ s-2002-20126.

5. Seifert L, Chollet D, Chatard JC. Kinematic changes during a 100-m front crawl: effects of performance level and gender. Med Sci Sports Exerc. 2007;39(10):17841793; doi: 10.1249/mss.0b013e3180f62f38.

6. Simbaña-Escobar D, Hellard P, Seifert L. Modelling stroking parameters in competitive sprint swimming: understanding inter- and intra-lap variability to as- 
A. Freudenheim, F. Madureira, U. Corrêa, Arm stroke timing in front crawl swim

sess pacing management. Hum Mov Sci. 2018;61:219230; doi: 10.1016/j.humov.2018.08.002.

7. Deschodt VJ, Arsac LM, Rouard AH. Relative contribution of arms and legs in humans to propulsion in 25-m sprint front-crawl swimming. Eur J Appl Physiol Occup Physiol. 1999;80(3):192-199; doi: 10.1007/ s004210050581.

8. Gourgoulis V, Boli A, Aggeloussis N, Toubekis A, Antoniou P, Kasimatis P, et al. The effect of leg kick on sprint front crawl swimming. J Sports Sci. 2014;32(3): 278-289; doi: 10.1080/02640414.2013.823224.

9. Morouço PG, Marinho DA, Izquierdo M, Neiva H, Marques MC. Relative contribution of arms and legs in $30 \mathrm{~s}$ fully tethered front crawl swimming. Biomed Res Int. 2015;2015:563206; doi: 10.1155/2015/563206.

10. Seifert L, Boulesteix L, Carter M, Chollet D. The spatial-temporal and coordinative structures in elite male 100-m front crawl swimmers. Int J Sports Med. 2005;26(4):286-293; doi: 10.1055/s-2004-821010.

11. Seifert L, Chollet D, Bardy BG. Effect of swimming velocity on arm coordination in the front crawl: a dynamic analysis. J Sports Sci. 2004;22(7):651-660; doi: 10.1080/02640410310001655787.

12. Chollet D, Pelayo P, Delaplace C, Tourny C, Sidney M. Stroking characteristic variations in the 100-m freestyle for male swimmers of differing skill. Percept Mot Skills. 1997;85(1):167-177; doi: 10.2466/pms.1997.85.1.167.

13. Hellard P, Dekerle J, Avalos M, Caudal N, Knopp M, Hausswirth C. Kinematic measures and stroke rate variability in elite female $200-\mathrm{m}$ swimmers in the four swimming techniques: Athens 2004 Olympic semifinalists and French National 2004 Championship semi-finalists. J Sports Sci. 2008;26(1):35-46; doi: 10.1080/02640410701332515.

14. Kjendlie P-L, Haljand R, Fjørtoft O, Stallman RK. Stroke frequency strategies of international and national swimmers in 100m races. Rev Port Cien Desp. 2006;6(Suppl. 2):52-54.

15. Corrêa UC, Benda RN, de Oliveira DL, Ugrinowitsch H, Freudenheim AM, Tani G. Different faces of variability in the adaptive process of motor skill learning. Nonlinear Dynamics Psychol Life Sci. 2015;19(4):465-487.

16. Barros JAC, Tani G, Corrêa UC. Effects of practice schedule and task specificity on the adaptive process of motor learning. Hum Mov Sci. 2017;55:196-210; doi: 10.1016/j.humov.2017.07.011.

17. Corrêa UC, Correia WR, Tani G. Towards the teaching of motor skills as a system of growing complexity. In: Koopmans M, Stamovlasis D (eds.), Complex dynamical systems in education: concepts, methods and applications. Cham: Springer; 2016; 93-103.

18. De Paula Pinheiro J, Marques PG, Tani G, Corrêa UC. Diversification of motor skills rely upon an optimal amount of variability of perceptive and motor task demands. Adapt Behav. 2015;23(2):83-96; doi: 10.1177/ 1059712315571369.
19. Tani G, Corrêa UC, Basso L, Benda RN, Ugrinowitsch H, Choshi K. An adaptive process model of motor learning: insights for the teaching of motor skills. Nonlinear Dynamics Psychol Life Sci. 2014;18(1):47-65.

20. Barabási A-L. Linked: the new science of networks. Cambridge: Perseus Books Group; 2002.

21. Kelso JAS, Engstrom DA. The complementary nature. Cambridge: MIT Press; 2006.

22. Salthe SN. Hierarchical structures. Axiomathes. 2012; 22(3):355-383; doi: 10.1007/s10516-012-9185-0.

23. Weiss PA. The living system: determination stratified. Stud Gen. 1969;22(4):361-400.

24. Weiss PA. The basic concept of hierarchic systems. In: Weiss PA (ed.), Hierarchically organized systems in theory and practice. New York: Hafner; 1971; 1-43.

25. Maglischo EW. Swimming fastest. Champaign: Human Kinetics; 2003.

26. Madureira F, Bastos FH, Corrêa UC, Rogel T, Freudenheim AM. Assessment of beginners' front-crawl stroke efficiency. Percept Mot Skills. 2012;115(1):300-308; doi: 10.2466/06.05.25.PMS.115.4.300-308.

27. Apolinário MR, Oliveira TAC, Ferreira LF, Basso L, Corrêa UC, Freudenheim AM. Effects of different breathing patterns on the performance and the arm stroke in the front crawl [in Portuguese]. Rev Bras Educ Fis Esporte. 2012;26(1):149-159; doi: 10.1590/S180755092012000100014.

28. De Oliveira TAC, Torriani-Pasin C, Silva SL, Denardi RA, Madureira F, Apolinário MR, et al. The spatiotemporal constraint on the swimmer's decisionmaking of turning. Motricidade. 2014;10(3):90-98; doi: 10.6063/motricidade.10(3).3052.

29. Bradshaw EJ, Maulder PS, Keogh JWL. Biological movement variability during the sprint start: performance enhancement or hindrance? Sports Biomech. 2007;6(3):246-260;doi:10.1080/14763140701489660.

30. Bland JM, Altman DG. Matching. BMJ. 1994;309(6962): 1128; doi: 10.1136/bmj.309.6962.1128.

31. Lampadari V, Thanopoulos V, Dopsaj M, Rozi G. Effects of age and gender in physiological responses, mechanics, and performance of master swimmers. Hum Mov. 2019;20(1):17-23; doi: 10.5114/hm.2019.79393.

32. Gentner DR. Timing of skilled motor performance: tests of the proportional duration model. Psychol Rev. 1987; 94(2):255-276; doi: 10.1037/0033-295X.94.2.255.

33. Heuer H. Invariant relative timing in motor-program theory. Adv Psychol. 1991;81:37-68; doi: 10.1016/ S0166-4115(08)60759-1.

34. Kelso JAS. Relative timing in brain and behavior: some observations about the generalized motor program and self-organized coordination dynamics. Hum Mov Sci. 1997;16(4):453-460; doi: 10.1016/S0167-9457(96)00 044-9.

35. Schmidt RA. The search for invariance in skilled movement behavior. Res Q Exerc Sport. 1985;56(2):188-200; doi: 10.1080/02701367.1985.10608457. 
36. Abbiss CR, Laursen PB. Describing and understanding pacing strategies during athletic competition. Sports Med. 2008;38(3):239-252; doi: 10.2165/00007256200838030-00004.

37. Freudenheim AM, Basso L, Filho EX, Madureira F, da Silva CGS, Manoel EJ. Temporal organization of stroke in the swimming crawl: beginners "versus" skilled [in Portuguese]. Rev Bras Cienc Mov. 2005;13(2):75-84. 